\title{
A SIMPLE, HIGH-THROUGHPUT METHOD TO DETECT PLASMODIUM FALCIPARUM SINGLE NUCLEOTIDE POLYMORPHISMS IN THE DIHYDROFOLATE REDUCTASE, DIHYDROPTEROATE SYNTHASE, AND $P$. FALCIPARUM CHLOROQUINE RESISTANCE TRANSPORTER GENES USING POLYMERASE CHAIN REACTION- AND ENZYME-LINKED IMMUNOSORBENT ASSAY-BASED TECHNOLOGY
}

\begin{abstract}
MICHAEL ALIFRANGIS, SONIA ENOSSE, RICHARD PEARCE, CHRIS DRAKELEY, CALLY ROPER, INSAF F. KHALIL, WATOKY MMM NKYA, ANITA M. RØNN, THOR G. THEANDER, AND IB C. BYGBJERG Centre for Medical Parasitology, Institute of Medical Microbiology and Immunology, and Institute of Public Health, University of Copenhagen, Copenhagen, Denmark; Department of Infectious Diseases, Copenhagen University Hospital, Copenhagen, Denmark; Instituto Nacional de Saúde, Ministério de Saúde, Maputo, Mozambique; Department of Infectious and Tropical Diseases, London School of Hygiene and Tropical Medicine, London, United Kingdom; Kilimanjaro Christian Medical Centre, Moshi, Tanzania; The Joint Malaria Programme, Moshi, Tanzania
\end{abstract}

Abstract. Single nucleotide polymorphisms (SNPs) in the Plasmodium falciparum dihydrofolate reductase (dhfr), and dihydropteroate synthetase $(d h p s)$, and chloroquine resistance transporter $(P f c r t)$ genes are used as molecular markers of $P$. falciparum resistance to sulfadoxine/pyrimethamine and chloroquine. However, to be a practical tool in the surveillance of drug resistance, simpler methods for high-throughput haplotyping are warranted. Here we describe a quick and simple technique that detects $d h f r$, dhps, and Pfcrt SNPs using polymerase chain reaction (PCR)- and enzyme-linked immunosorbent assay (ELISA)-based technology. Biotinylated PCR products of dhfr, dhps, or Pfcrt were captured on streptavidin-coated microtiter plates and sequence-specific oligonucleotide probes (SSOPs) were hybridized with the PCR products. A stringent washing procedure enabled detection of remaining bound SSOPs and distinguished between the SNPs of $d h f r, d h p s$, and Pfcrt with high specificity. The SSOP-ELISA compared well with a standard PCR-restriction fragment length polymorphism procedure, and gave identical positive results in more than $90 \%$ of the $P$. falciparum slide-positive samples tested. The SSOP-ELISA of all $d h f r$, $d h p s$, or Pfcrt SNPs on 88 samples can be performed in a single day and provides quick and reproducible results. The system can potentially be modified to detect SNPs in other genes.

\section{INTRODUCTION}

The sulfadoxine-pyrimethamine (SP) drug combination is widely used in malaria-endemic countries in sub-Saharan Africa as a first-line drug to treat uncomplicated Plasmodium falciparum malaria and has replaced chloroquine (CQ) in many endemic countries due to widespread resistance. Despite the relatively recent introduction of SP into, for example, east Africa, alarming levels of $P$. falciparum treatment failures to SP have been recorded in the region. ${ }^{1}$ The continuous monitoring of resistance to SP and other antimalarial drugs is therefore of major importance for rational decisions regarding future treatment guidelines.

Combinations of single nucleotide polymorphisms (SNPs) in the dihydrofolate reductase $(d h f r)$ gene at codons 51, 59, 108 and 164 and the dihydropteroate synthetase (dhps) gene at codons 436, 437, 540, 581, and 613 correlate with $P$. falciparum resistance to SP in vivo. ${ }^{2-4}$ Primarily, mutations in codons 51, 59, and 108 of $d h f r$ coupled with codons 437 and 540 of dhps have been associated with a high risk of SP treatment failure. ${ }^{2,3}$ For CQ, a single key mutation at position 76 of the $P$. falciparum chloroquine resistance transporter (Pfcrt) gene has been linked to CQ resistance. ${ }^{5-7}$ Thus, increasing problems with drug resistance are reflected in the frequency of mutations in these genes and longitudinal monitoring of mutations in the parasite population can be used in large-scale surveillance of drug resistance..$^{2,8,9}$

Currently, the most commonly used method to identify SNPs in dhfr/dhps and Pfcrt is a nested polymerase chain reaction (PCR) followed by restriction fragment length polymorphism (RFLP) analysis. ${ }^{10}$ This method is reliable, but not suitable for high-throughput screening. Furthermore, the re- sults are constrained to individual analysis of each SNP and interpretation is complicated by the occurrence of multiclonal $P$. falciparum infections. ${ }^{11}$

Analyses of haplotypes detecting combinations of SNPs in one gene may be more suitable and biologically sound than measuring the prevalence of SNPs when assessing SP and CQ resistance at a population level, ${ }^{12}$ and alternatives to the PCR-RFLP method have been developed. Abdel-Muhsin and others described a dot-blot method for the detection of a limited number of SNPs in $d h f r$ using radiolabeled sequencespecific oligonucleotide probes (SSOPs) post-PCR. ${ }^{13}$ Mehlotra and others developed a similar system for the detection of Pfcrt haplotypes at c72-76 using fluorescein isothiocyanatelabeled SSOPs. ${ }^{14}$ Pearce and others described a highthroughput PCR SSOP-dot blot method detecting all known SNPs of $d h f r$ and $d h p s$ using digoxigenin-labeled probes and high stringency washing with tetra-methyl-ammonium chloride (TMAC), enabling similar stringent washing procedures for almost all the SSOPs, simplifying the methodology significantly. $^{12}$

Here, we describe a dot blot-based technique where the SNPs are visualized in an enzyme-linked immunosorbent assay (ELISA) system. The technique is simple and allows laboratories with limited laboratory facilities to process a large number of samples. The methodology is applicable regardless of the gene or organism under investigation, and the technique can be applied as a general tool for the detection of SNPs.

\section{MATERIALS AND METHODS}

Plasmodium falciparum isolates and samples from patients. Five $P$. falciparum laboratory isolates (3D7, FCR3, 
7G8, K1, and DD2) were used to verify the specificity of the technique and applied as a standardized control panel in subsequent experiments. Additionally, samples from three $P$. falciparum-infected patients were used in the panel since they were found to express the 436/437AA, 436/437AG, and 540E genotypes of dhps. Almost all known SNPs of $d h f r, d h p s$, and Pfcrt (codons 72-76) are represented in this panel of parasites.

To test the validity of the SSOP-ELISA technique, we compared it to the dot blot method described by Pearce and others ${ }^{12}$ and the PCR-RFLP method described by Duraisingh and others ${ }^{10}$ using 84 samples collected on filter paper at the Tanganyika Planting Company (TPC) sugarcane plantation in Moshi, Tanzania. All samples were collected after informed consent was obtained from the patients by trained clinicians at the TPC sugar plantation hospital. Ethical permission for the study was obtained from the Tanzanian National Institute of Medical Research ethics committee and the London School of Hygiene and Tropical Medicine. The samples were for comparison and validation of the methods and the details of the patients will be described elsewhere (Drakeley $\mathrm{C}$ and others, unpublished data).

Extraction of DNA from parasites in culture and from blood samples on filter paper. Parasite isolates were grown in vitro and approximately $2.5 \times 10^{8}$ parasites were purified by magnetic activated cell sorting. ${ }^{15}$ The purified parasite cultures were extracted with phenol/chloroform and precipitated with ethanol as described by Sambrook and others. ${ }^{16}$ The resulting DNA pellet was resuspended in $30 \mu \mathrm{L}$ of water. The 3D7 isolate was used to determine the sensitivity of the SSOP-ELISA. DNA was extracted from 250,000 3D7 parasites and resuspended in $50 \mu \mathrm{L}$ of water. The sample was diluted 10-fold to a concentration corresponding to one parasite genome $/ \mu \mathrm{L}$. Extraction of DNA from bloodspots on filter paper was carried out by the Chelex-100 method described by Wooden and others ${ }^{17}$ with some modifications described by Pearce and others. ${ }^{12}$

Polymerase chain reactions for dhfrldhps and Pfcrt. A nested PCR described by Pearce and others ${ }^{12}$ was used to amplify fragments of the $d h f r$ and $d h p s$ genes. A nested PCR described by Djimde and others ${ }^{18}$ (described in detail at http://medschool.umaryland.edu/CVD/nejm2001djimde.htm) was used to amplify fragments of the Pfcrt gene. The only modification in our procedures was that the M9, R/, and TCRD2 primers for the $d h f r$, dhps, and Pfcrt nested PCRs were biotinylated at the $5^{\prime}$-end by the supplier (MWG Biotech, Riskov, Denmark). The 20- $\mu \mathrm{L}$ dhfr/dhps outer PCR mixture consisted of $0.3 \mathrm{mM}$ of each dNTP, $0.25 \mu \mathrm{M}$ of either primer set M1/M7 (dhfr) or N1/N2 (dhps), one unit of DNA HotStart polymerase (Qiagen, Albertslund, Denmark), buffer containing $1.5 \mathrm{mM} \mathrm{MgCl}_{2}$, as recommended by the manufacturer (Qiagen), and $1 \mu \mathrm{L}$ of extracted DNA.

Prior to use of the outer $d h f r$ PCR products for the nested reactions, the products were diluted 1:20 in distilled water in a PCR plate and $1 \mu \mathrm{L}$ of these dilutions or the outer dhps PCR products were used in the nested PCRs. The nested $d h f r$ and dhps PCR reaction mixture was the same as the outer PCR mixture using primer sets M3b/M9 and R2/R/ for the $d h f r$ and dhps PCR, respectively. The outer and nested $d h f r /$ dhps PCR conditions were as previously described. ${ }^{12}$

The 20- $\mu \mathrm{L}$ Pfcrt outer PCR mixture consisted of $0.2 \mathrm{mM}$ of each dNTP, $1 \mu \mathrm{M}$ of the primer set TCRP1/TCRP2, 1.25 units of DNA Qiagen HotStart polymerase, buffer containing 2.5
$\mathrm{mM} \mathrm{MgCl}_{2}$, and $1 \mu \mathrm{L}$ of extracted DNA. The reaction mixture of the nested Pfcrt PCR was identical to that of the outer PCR and the primer set TCRD1/TCRD2 was used. The conditions of the outer and nested Pfcrt PCR were as previously described. $^{18}$

Amplifications were performed in 96-well PCR plates and the reaction mixture was overlaid with one drop of mineral oil. The nested PCR products were confirmed by electrophoresis on a $1.5 \%$ agarose gel along with a set of controls.

SSOP-ELISA. The ELISA plates (Maxisorp; Nunc, Roskilde, Denmark) were coated with streptavidin in phosphatebuffered saline (PBS) $(1 \mu \mathrm{g} / \mathrm{mL})$, covered,and left overnight at $4{ }^{\circ} \mathrm{C}$. The plates could be kept for at least two weeks at $4^{\circ} \mathrm{C}$ without loss of reactivity. Prior to use, the plates were washed three times in washing buffer $(1 \times$ PBS containing $0.05 \%$ Tween 20).

The nested PCR products were diluted 1:10 in water in a 96-well PCR plate, denatured at $95^{\circ} \mathrm{C}$ for 5 minutes, and immediately thereafter cooled to $4^{\circ} \mathrm{C}$ until use. One hundred microliters of cold dilution buffer $(1 \times$ PBS with $0.05 \%$ Tween $20)$ and $2 \mu \mathrm{L}$ of the diluted PCR products was then added to each well of the ELISA plate. Replicate ELISA plates were made to enable simultaneous probing with SSOPs targeting the full panel of $d h f r$, dhps, and Pfcrt SNP/haplotypes. The plates were incubated at room temperature for one hour and washed three times in washing buffer. The 3 '-end digoxigenin-conjugated SSOPs (MWG Biotech) were diluted in tetramethyl ammonium chloride (TMAC; Sigma Aldrich Chemie, Seelze, Germany) solution (3 M TMAC, $50 \mathrm{mM}$ Tris, $\mathrm{pH} 8.0$, $0.1 \%$ sodium dodecyl sulfate, 2 mM EDTA, pH 8.0), heated to $53^{\circ} \mathrm{C}$, and $100 \mu \mathrm{L}$ was then added to each well at the concentrations indicated in Table 1 . The plates were then incubated in a hybridization oven (AH Diagnostics, Aarhus, Denmark) at $53^{\circ} \mathrm{C}$ on a shaking device for one hour and washed three times in washing buffer. This was followed by two rounds of washing and incubation (10 minutes per round) in TMAC solution at the temperatures indicated in Table 1. To remove TMAC, the plates were then washed three times in washing buffer and peroxidase-conjugated anti-digoxigenin antibody in dilution buffer $(1: 1,000)$ (Roche Diagnostics, Mannheim, Germany) was added to each well. After incubation for one hour at room temperature, the plates were washed three times in washing buffer and an $o$-phenylenediamine solution of $1.5 \mathrm{mg} / \mathrm{mL}$ of 1,2-phenyldiamine dihydrochloride (Dako, Glostrup, Denmark) dissolved in water containing $0.015 \% \mathrm{H}_{2} \mathrm{O}_{2}$ was added to the plates. After 30 minutes, the reaction was stopped by adding $1.25 \mathrm{M}$ $\mathrm{H}_{2} \mathrm{SO}_{4}$ and the optical density (OD) at $492 \mathrm{~nm}$ was measured in an ELISA reader. A flow chart of the method is shown in Figure 1.

SSOP-dot blot. The SSOP-dot blot was performed as described by Pearce and others ${ }^{12}$ and Conway and others ${ }^{19}$ with a few modifications: Two microliters of nested PCR products were spotted onto nylon membranes (Roche Diagnostics) and cross-linked to them at $120^{\circ} \mathrm{C}$ for 30 minutes. After SSOP incubation and two high-stringency washings with TMAC (10 minutes per wash) $\left(58^{\circ} \mathrm{C}\right.$ for all probes, except for c59 and c108, which were washed at $\left.62^{\circ} \mathrm{C}\right)$, the membranes were washed in maleic acid washing buffer $(0.1 \mathrm{M}$ maleic acid, 0.15 $\mathrm{M} \mathrm{NaCl}, 0.3 \%$ Tween $20, \mathrm{pH} 7.5)$ for 30 minutes. The membranes were placed in blocking buffer $(0.1 \mathrm{M}$ maleic acid, 0.15 $\mathrm{M} \mathrm{NaCl}, 10 \%$ Tween $20,1 \%$ milk powder) for 30 minutes. 
TABLE 1

Distribution of the probes used for the $d h f r$, dhps, and Pfcrt ELISA haplotyping assay*

\begin{tabular}{|c|c|c|c|c|}
\hline SSOP & SSOP sequence $\dagger$ & $\begin{array}{c}\text { Probe } \\
\text { concentrationł }\end{array}$ & $\begin{array}{l}\text { Washing } \\
\text { temperature§ }\end{array}$ & Isolate(s)氏ा \\
\hline$d h f r$ & & \multirow[t]{3}{*}{$\mathrm{nM}$} & \multirow[t]{3}{*}{$\left(\mathrm{C}^{\circ}\right)$} & \\
\hline $50 / 51 \mathrm{CN}$ & TGG AAA TGT AAT TCC CTA & & & 3D7, FCR3, K1 \\
\hline $50 / 51 \mathrm{CI}$ & TGG AAA TGT ATT TCC CTA & & & $\mathrm{DD} 2$ \\
\hline $50 / 51 \mathrm{CN} 2$ & TGG AAA TGT AAC TCC CTA & \multirow[t]{4}{*}{20} & \multirow[t]{4}{*}{58} & - \\
\hline $50 / 51 \mathrm{RN}$ & TGG AAA CGT AAT TCC CTA & & & - \\
\hline $50 / 51 \mathrm{RN} 2$ & TGG AAA CGT AAC TCC CTA & & & - \\
\hline 50/51RI & TGG AAA CGT ATT TCC CTA & & & - \\
\hline $59 \mathrm{C}$ & AAT ATT TTT GTG CAG TTA & \multirow[t]{2}{*}{4} & \multirow[t]{2}{*}{60} & 3D7, FCR3 \\
\hline $59 \mathrm{R}$ & AAT ATT TTC GTG CAG TTA & & & DD2, K1 \\
\hline $108 \mathrm{~S}$ & A AGA ACA AGC TGG GAA AG & \multirow{3}{*}{4} & \multirow{3}{*}{62} & 3D7 \\
\hline $108 \mathrm{~N}$ & A AGA ACA AAC TGG GAA AG & & & $\mathrm{DD} 2, \mathrm{~K} 1$ \\
\hline $108 \mathrm{~T}$ & A AGA ACA ACC TGG GAA AG & & & FCR3 \\
\hline 164I & GT TTT ATT ATA GGA GGT T & \multirow[t]{2}{*}{4} & \multirow[t]{2}{*}{60} & - \\
\hline $164 \mathrm{~L}$ & GT TTT ATT TTA GGA GGT T & & & - \\
\hline \multicolumn{5}{|l|}{ dhps } \\
\hline 436/437AA & GAA TCC GCT GCT CCT TTT & \multirow{7}{*}{4} & \multirow{7}{*}{60} & DK1 \\
\hline 436/437AG & GAA TCC GCT GGT CCT TTT & & & DK4 \\
\hline 436/437SA & GAA TCC TCT GCT CCT TTT & & & FCR3 \\
\hline 436/437SG & GAA TCC TCT GGT CCT TTT & & & 3D7, K1 \\
\hline 436/437FA & GAA TCC TTT GCT CCT TTT & & & - \\
\hline 436/437FG & GAA TCC TTT GGT CCT TTT & & & DD2 \\
\hline 436/437CA & GAA TCC TGT GCT CCT TTT & & & - \\
\hline $540 \mathrm{~K}$ & ACA ATG GAT AAA CTA ACA & \multirow[b]{2}{*}{4} & \multirow{2}{*}{60} & 3D7, FCR3, DD2, K1, \\
\hline $540 \mathrm{E}$ & ACA ATG GAT GAA CTA ACA & & & $\begin{array}{c}\text { DK1, DK4 } \\
\text { SP70 }\end{array}$ \\
\hline $581 \mathrm{~A}$ & A GGA TTT GCG AAG AAA CA & \multirow[b]{2}{*}{4} & \multirow[b]{2}{*}{60} & 3D7, FCR3, DD2, DK1, \\
\hline $581 \mathrm{G}$ & A GGA TTT GGG AAG AAA CA & & & $\begin{array}{c}\mathrm{DK} 4, \mathrm{SP} 70 \\
\mathrm{~K} 1\end{array}$ \\
\hline $613 \mathrm{~A}$ & GA TTT ATT GCC CAT TGC & \multirow[t]{3}{*}{4} & \multirow[t]{3}{*}{60} & $\begin{array}{l}\text { 3D7, FCR3, K1, DK1, } \\
\text { DK4, SP70 }\end{array}$ \\
\hline $613 \mathrm{~S}$ & GA TTT ATT TCC CAT TGC & & & DD2 \\
\hline $613 \mathrm{~T}$ & GA TTT ATT ACC CAT TGC & & & - \\
\hline \multicolumn{5}{|l|}{ Pfcrt c72-76 } \\
\hline CVMNK & TA TGT GTA ATG AAT AAA A & \multirow{5}{*}{4} & \multirow{5}{*}{60} & 3D7 \\
\hline CVIET & TA TGT GTA ATT GAA ACA A & & & FCR3 \\
\hline SVMNT & TA AGT GTA ATG AAT ACA A & & & 7G8 \\
\hline CVIEK & TA TGT GTA ATT GAA AAA A & & & - \\
\hline $\mathrm{S}_{2} \mathrm{VMNT}$ & TA TCT GTA ATG AAT ACA A & & & - \\
\hline
\end{tabular}

Alkaline phosphatase-conjugated anti-digoxigenin Fab fragments (Roche Diagnostics) diluted 1:5,000 in maleic acid washing buffer was added to the membranes and the membranes were incubated for 30 minutes. The membranes were then washed twice (15 minutes per wash) in maleic acid washing buffer and semi-dried. Finally, positive reactions were detected with CDP-star substrate (Roche Diagnostics) and exposure on Hyperfilm-ECL (Amersham, Bucks, United Kingdom).

PCR-RFLP assay. The outer $d h f r$ and $d h p s$ PCR products produced as described earlier were used in the nested $d h f r$ and dhps PCR-RFLP assays; otherwise, the nested primers and conditions described by Duraisingh and others ${ }^{10}$ was used. The RFLP was performed using restriction enzymes and buffers obtained from New England Biolabs (Medinova, Glostrup, Denmark). The digested products were visualized by electrophoresis on 2-2.5\% Metaphor agarose gels (Medi- nova) and analyzed using Kodak (Rochester, NY) 1D software version 3.5.3.

Scoring of ELISA and dot-blot data. The OD values of positive and negative controls varied between experiments, although only rarely compromising specificity. The variation was possibly due to marginal differences in the strength of the probe binding and washing force during the high-stringency washes. Thus, no fixed threshold value could be specified and for each SNP test, a simple analysis of the positive and negative control samples was performed to set a threshold for positivity. For each SNP analyses, parasite samples were categorized into single, mixed but with one SNP in majority or mixed infections as follows: Infections were considered to be of single genotype when only one SNP was present at OD values above the threshold of positivity. Samples were considered to be mixed but containing a majority SNP genotype 
SSOP-ELISA PROCEDURE

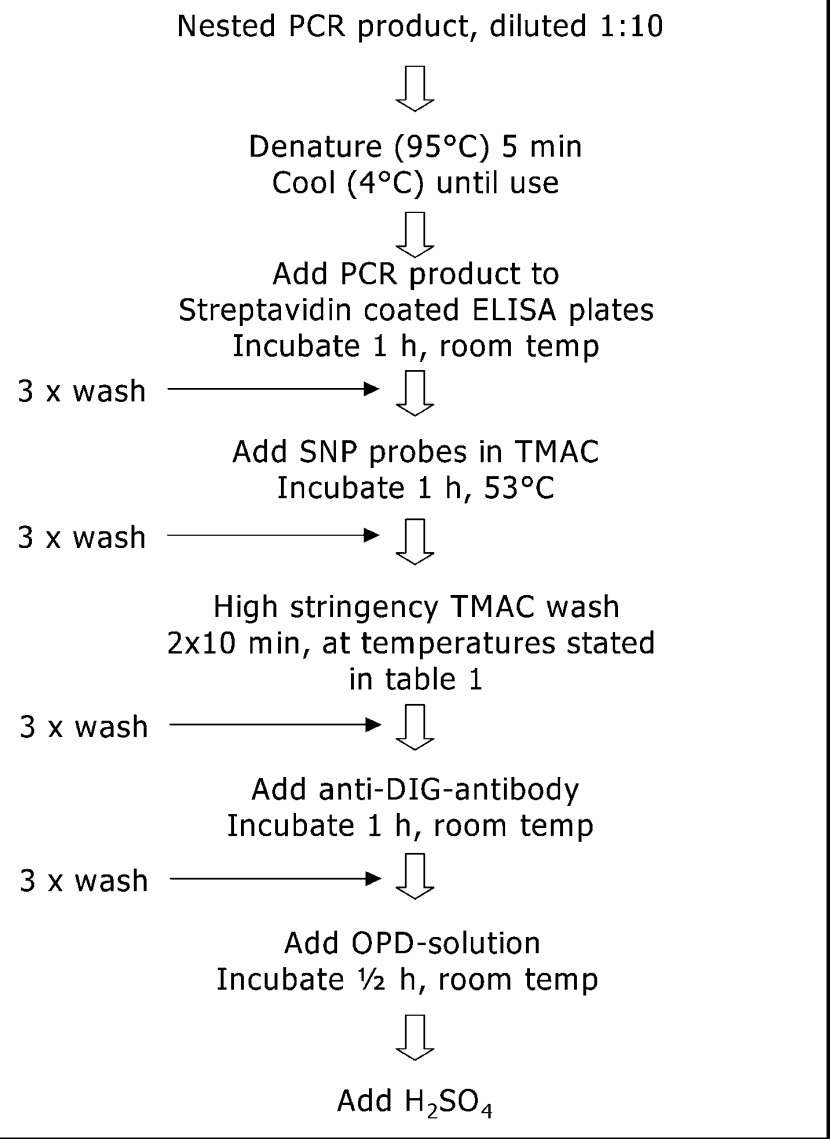

Figure 1. Flow chart of the dihydrofolate reductase, dihydropteroate synthase, and Plasmodium falciparum chloroquine resistance transporter sequence-specific oligonucleotide probe-enzymelinked immunosorbent assay (SSOP-ELISA) method. PCR = polymerase chain reaction; SNP = single nucleotide polymorphism; TMAC = tetra-methyl ammonium chloride; DIG = digoxigenin; $\mathrm{OPD}=o$-phenylenediamine.

when the OD value of the weakly reacting SSOP was less than half the OD value of the strongly reacting SSOP. Conversely, if the OD value of the weakly reacting SSOP was higher than half the OD value of the strongly reacting SSOP, the infection was categorized as mixed with no dominant genotype. For samples that contained infections categorized as single or mixed with a dominant SNP type at all analyzed codons, results were combined to construct haplotypes.

The manual scoring of the dot-blot results was performed on a light table. The definition of single, majority, or mixed infections for each SNP and combined as haplotypes was performed with a similar methodology as described earlier.

\section{RESULTS}

Specificity and sensitivity of SSOP-ELISA haplotyping assay. To test the specificity of the $d h f r$ SSOP-ELISA, the four laboratory isolates 3D7, FCR3, DD2, and K1 known to carry different $d h f r$ haplotypes were tested. Figure 2 show that the assay correctly identified the SNPs of the different isolates using SSOPs for 50/51CN, 50/51CI, 59C, 59R, 108S, 108N, and 108T. The OD values of the positive reactions obtained with
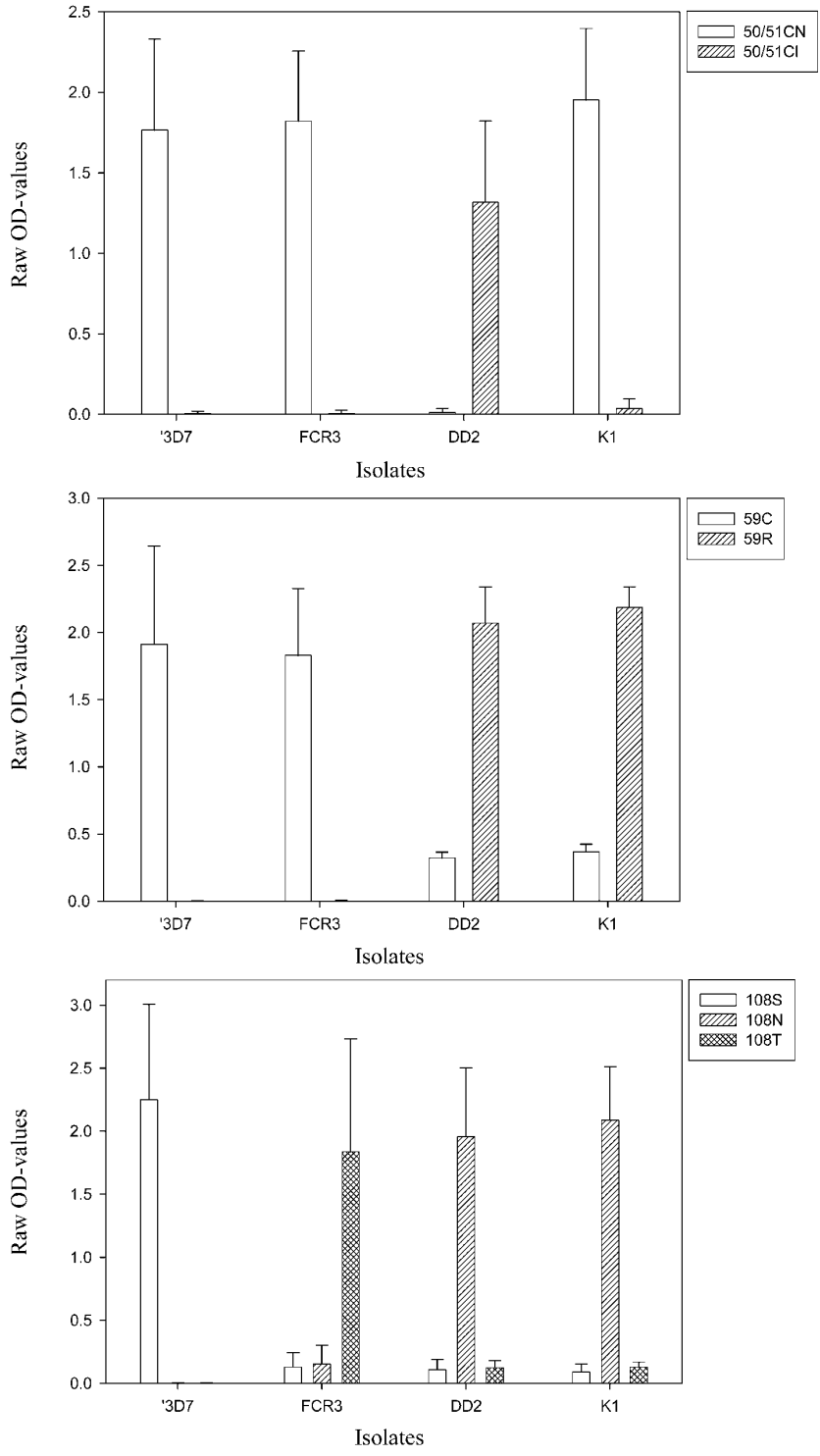

FIGURE 2. Specificity of the dihydrofolate reductase (dhfr) sequence-specific oligonucleotide probe-enzyme-linked immunosorbent assay. Reactivity of samples from different parasite isolates (3D7, FCR3, DD2, and K1) using probes $(50 / 51 \mathrm{CN}, 50 / 51 \mathrm{CI}, 59 \mathrm{C}$, $59 \mathrm{R}, 108 \mathrm{~S}, 108 \mathrm{~N}$, and $108 \mathrm{~T})$ targeting single nucleotide polymorphisms in $d h f r$. Optical density (OD) values are the means and $95 \%$ confidence intervals of six independent experiments. The results are in concordance with published genotypes of the tested isolates (Table 1).

specific probes were markedly higher than the OD values obtained when using unspecific probes. The difference in OD value between the threshold of positivity and the positive reactions $(\Delta \mathrm{OD})$ was always greater than 1 . To assess the specificity for most of the SSOPs in the dhps SSOP-ELISA, the four laboratory isolates and three additional parasite isolates from malaria patients were tested using SSOPs for 436/ 437AA, 436/437AG, 436/437SA, 436/437SG, 436/437FG and for $540 \mathrm{~K}, 540 \mathrm{E}, 581 \mathrm{~A}, 581 \mathrm{G}, 613 \mathrm{~A}$, and $613 \mathrm{~S}$ (Figure 3 ). The assay correctly identified the SNPs of the different isolates. The threshold of positivity and the $\triangle$ OD vary between SNPs and between consecutive experiments; however, the $\Delta \mathrm{ODs}$ for the different SNPs were at least 1. Testing of the remaining SNPs of $d h f r$ (variants at c50/51 and 164L) and dhps (436/ 

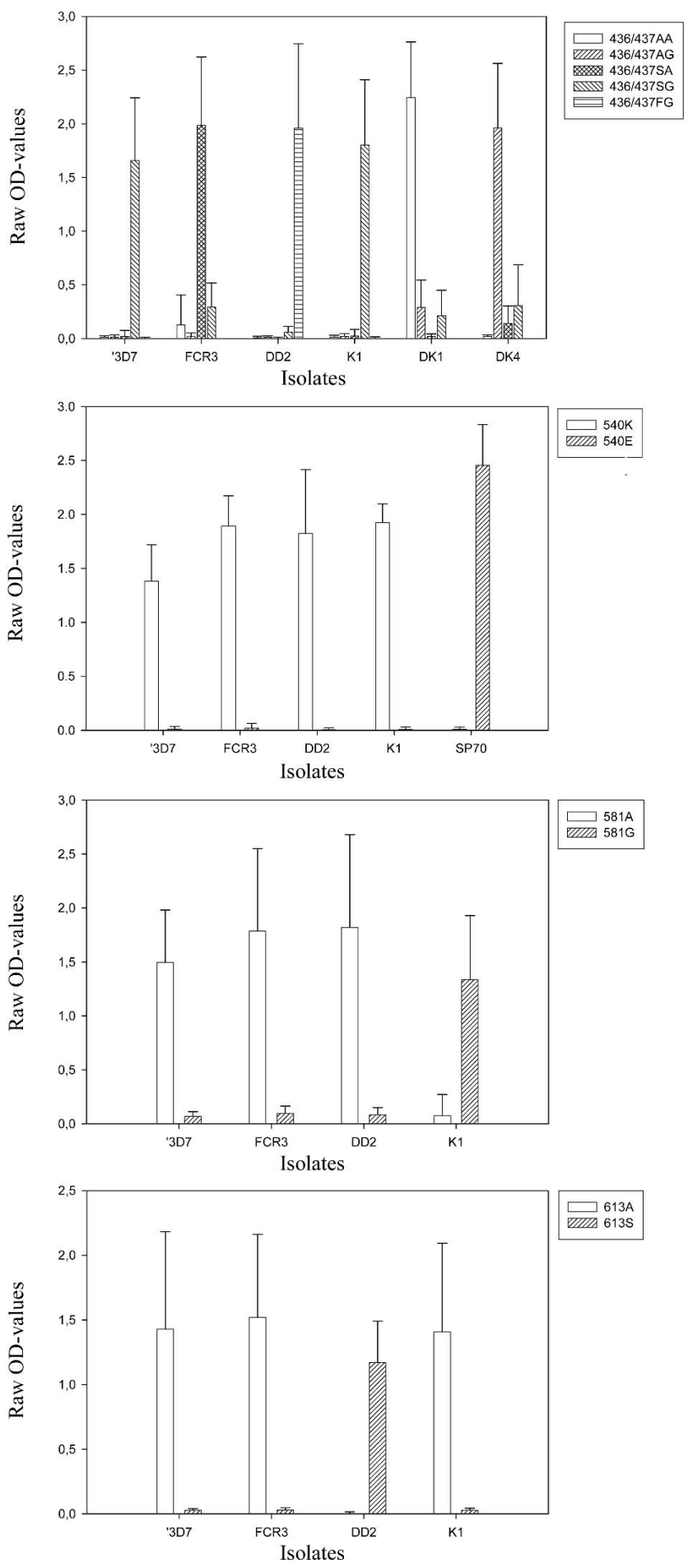

FIGURE 3. Specificity of the dihydropteroate synthase (dhps) sequence-specific oligonucleotide probe-enzyme-linked immunosorbent assay. Reactivity of samples from different parasite isolates (3D7, FCR3, DD2, and K1) and parasites from three patient samples (DK1, DK4, and SP70) using the probes 436/437AA, 436/437AG, 436/437SA, 436/437SG, 436/437FG, 540K, 540E, 581A, 581G, 613A, and $613 \mathrm{~S}$ targeting single nucleotide polymorphisms in $\mathrm{dhps}$. Optical density (OD) values are the means and $95 \%$ confidence intervals of six independent experiments. The results are in concordance with published genotypes of the tested isolates (Table 1).

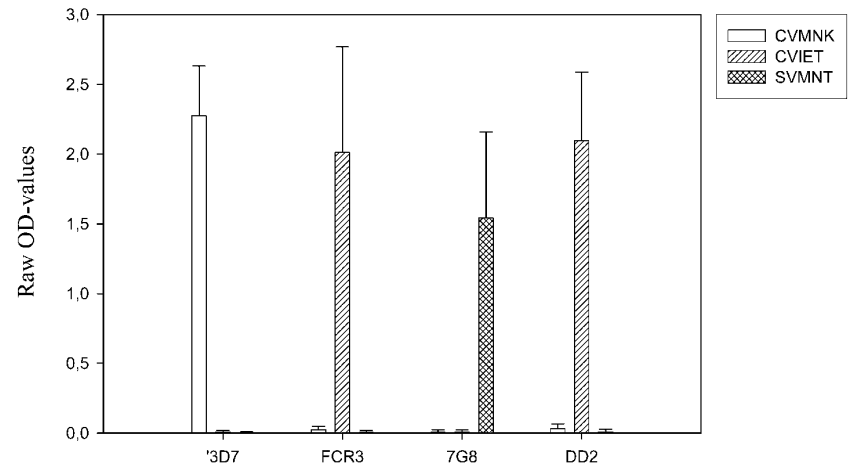

FIgURE 4. Specificity of the Plasmodium falciparum chloroquine resistance transporter $(P f c r t)$ sequence-specific oligonucleotide probe-enzyme-linked immunosorbent assay. Reactivity of samples from different parasite isolates (3D7, FCR3, 7G8, and DD2) using the probes CVMNK, CVIET, and SVMNT targeting haplotypes at c72-76 in Pfcrt. Optical density (OD) values are means and $95 \%$ confidence intervals of four independent experiments. The results are in concordance with published genotypes of the tested isolates (Table 1)

437CA, 436/437FA, and 613T) were done, but are not shown since positive controls for these SNPs were unavailable.

The specificity of the Pfcrt SSOP-ELISA was assessed using the 3D7, FCR3, 7G8, and DD2 laboratory isolates. Figure 4 show that the assay correctly identified the c72-76 haplotypes of the different isolates using SSOPs for CVMNK, CVIET, and SVMNK. The $\triangle \mathrm{OD}$ was at least 1 . Finally, the sensitivity of the SSOP-ELISA was determined using a 10fold dilution series of 3D7 with the $d h f r$ c108 SSOP-ELISA as standard. The results indicated that the SSOP-ELISA was capable of detecting DNA from one parasite genome.

SSOP-ELISA versus SSOP-dot blot haplotype detection. A comparison of results between the SSOP-ELISA technique and the published SSOP-dot blot method was made by comparing results obtained using 84 samples from $P$. falciparum slide-positive Tanzanian patients. For the detection of SNPs in $d h f r$, the analysis was performed by comparing combined haplotype data for c50/51, c59, and c108. Only marginal differences were observed between the two methods (Table 2) and identical positive results were obtained for 74 of 80 samples $(92.5 \%)$. The dhps haplotypes were constructed based on analysis of SNPs at 436/437 and c540. The two methods gave identical positive results for 65 of 69 samples (94.2\%) (Table 2). One sample was found to be AAK by dot blot, but SGE by SSOP-ELISA; however, this sample had the SGE haplotype as a minority infection by dot-blot.

SSOP-ELISA versus the RFLP method. The samples were also used to compare results obtained by the SSOP-ELISA and the RFLP method. The c50 dhfr polymorphism was not analyzed by RFLP because the SSOP-ELISA and SSOP-dot blot results indicated that only the c50C was prevalent in the area. There was only marginal disagreement between the two methods in the analysis of the $d h f r$ haplotype and identical positive results were obtained for 74 of 78 samples $(94.9 \%)$ (Table 3). One sample was found to be CIRN by RFLP but CNRN by SSOP-ELISA; however, the SSOP-ELISA analysis showed that CIRN was present as a minority haplotype. For the dhps haplotypes (Table 3 ), identical positive results were obtained in 49 of 54 samples $(90.7 \%)$. However, some differences were encountered. Seventeen samples were found to be 
TABLE 2

Comparison of results obtained with SSOP-ELISA and SSOP-dot blot targeting SNPs in dhfr (top) and dhps (bottom) using DNA extracted from 84 malaria patients*

\begin{tabular}{lccccccc}
\hline & \multicolumn{5}{c}{ Dot-blot } \\
\cline { 2 - 7 } ELISA & CNCS & CNRS & CNRN & CICN & CIRN & Mixed & Neg \\
\hline CNCS & 2 & & & & & & \\
CNRS & & & 3 & & & 3 & \\
CNRN & & & 3 & & & \\
CICN & & & & 9 & 56 & & \\
CIRN & & 1 & & & 2 & 4 & 1 \\
Mixed & & & & & & & 3 \\
Neg & & & & & &
\end{tabular}

\begin{tabular}{lcccccc}
\hline & \multicolumn{5}{c}{ Dot-blot } \\
\cline { 2 - 6 } ELISA & AAK & SAE & SAK & SGE & Mixed & Neg \\
\hline AAK & 2 & & & & & \\
SAE & & & 14 & & 1 & 1 \\
SAK & 1 & & & 45 & & 1 \\
SGE & 1 & & 1 & 1 & 4 & 7 \\
Mixed & & 1 & 2 & 3 & & 7
\end{tabular}

$*$ SNPs $=$ single nucleotide polymorphisms; Mixed $=$ mixed haplotype infections at one or more codons where a clear majority haplotype by one or both methods could not be or more codons where a clear majority haplotype by one or both methods could not be efinated. Neg ner ten. The types. The construction or $\mathrm{N}$ ) for $d h f r$ and c436/437 (AA, SA, or SG) and c540 (K or E) for dhps.

negative at one or more codons by RFLP and positive by SSOP-ELISA, while nine samples were negative at one or more codons by SSOP-ELISA and positive by RFLP. The detailed $d h f r$ and dhps genotype distribution by SSOPELISA of the patient samples is available as Appendix 1 at http://www.cmp.dk/SSOP-ELISA/TPC.pdf

\section{DISCUSSION}

This paper describes a new method to detect SNPs and shows that it can be adapted to evaluate SNPs in genes associated with resistance to two of the most widely used antimalarial drugs. The new SSOP-ELISA based method de-

TABLE 2

Comparison of results obtained with SSOP-ELISA and RFLP targeting SNPs in $d h f r$ (top) and dhps (bottom) using DNA extracted from 84 malaria patients*

\begin{tabular}{lcccccc}
\hline & \multicolumn{5}{c}{ RFLP } \\
\cline { 2 - 7 } ELISA & CNCS & CNRN & CICN & CIRN & Mixed & Neg \\
\hline CNCS & 2 & & & 1 & 1 & 1 \\
CNRN & & 3 & & 1 & & \\
CICN & & & 9 & 53 & 1 & 2 \\
CIRN & & & & 1 & 7 & 3 \\
Mixed & & & & & & 3 \\
Neg & & & &
\end{tabular}

\begin{tabular}{lccrcr}
\hline & \multicolumn{5}{c}{ RFLP } \\
\cline { 2 - 5 } ELISA & AAK & SAK & SGE & Mixed & Neg \\
\hline AAK & 2 & & & 2 & 3 \\
SAK & & 11 & 34 & 2 & 11 \\
SGE & & & 1 & 2 & 3 \\
Mixed & & 5 & 2 & 2 & 4 \\
Neg & & 5
\end{tabular}

*SNPs = single nucleotide polymorphisms; RFLP $=$ restriction fragment length polymorphism; Mixed $=$ mixed haplotype infections at one or more codons where a clear majority haplotype by one or both methods could not be elucidated. Neg = negative samples in one or more codons by one or both methods. For definitions of other abbreviations, see Table 1. The SNPs results are combined into haplotypes. The construction of haplotypes involved c50/51 (CN or CI), c59 (C or R), and c108 ( $\mathrm{S}$ or $\mathrm{N}$ ) for $d h f r$ and c436/437 (AA, SA or $\mathrm{SG}$ ) and c540 (K or $\mathrm{E})$ for dhps. scribed provides a simple high-throughput system that is likely to be of use in areas where there is only access to relatively limited laboratory facilities.

The methodology uses an outer and nested PCR for $d h f r$ and dhps amplification step almost identical to that described previously, ${ }^{12}$ except for the inclusion of one biotinylated primer in each nested PCR. The nested PCRs are performed as single nested PCRs for each gene in contrast to several needed for the PCR-RFLP method, simplifying the procedure and reducing the cost. This is because the PCR-RFLP method is sometimes hampered by the dependency of recognizable restriction sites in the sequence surrounding a SNP in question. Thus for this method, it is often necessary to design additional PCRs with modifications into one of the primers to provide a suitable restriction site. For the PCR-ELISAs, SSOP probes were either based on published probe sequences $^{12}$ or simply designed by targeting 18 basepairs surrounding the SNP in question, and it was generally not complicated to optimize the performance of the probes in the assay.

The high-throughput of the SSOP-ELISA method compared with the RFLP method is an important advantage. We used a panel of $P$. falciparum isolates with known SNPs to test the specificity of SSOP-ELISA system. For all the tested SNPs in $d h f r$, dhps, and Pfcrt genes, the signal-to-noise difference $(\Delta \mathrm{OD})$ was greater than 1 . The resulting OD values varied markedly between experiments. This might be due to probe hybridization efficiency, the TMAC stringency washing step, and marginal differences in the extremely sensitive nested PCR. However, we seldomly observed that the $\Delta \mathrm{OD}$ was less than 1 and in that case, the experiment was repeated. The high stringency TMAC washing involved three different temperatures for the $d h f r$ SNPs. It was possible to perform the stringency washing at $60^{\circ} \mathrm{C}$ for all the codons, although lower $\Delta$ ODs were encountered for the c108 SNPs and the c50/51 SNPs.

A high degree of concordance was observed when comparing the new SSOP-ELISA with the existing PCR-RFLP method, probably the most widely used of current methodologies. The SSOP-ELISA haplotyping has the advantage that it identifies the most abundant SNPs through its exact measures of OD, rather than detecting the more general mixed genotype infections alone. The samples used to compare the methods were mainly single genotype infections due to low malaria transmission in the area (Drakeley $\mathrm{C}$, unpublished data). Thus, this advantage was not seen frequently. However, to detect alleles in mixed infections that are present in very low quantities, none of these methods may be suitable. However, an alternative method used by Hastings and others has the capacity to detect these. ${ }^{20}$

As expected, the detection of SNPs in $d h f r$ and $d h p s$ by dot blotting and SSOP-ELISA gave generally similar results and both methods can be applied for high-throughput haplotyping of $d h f r$ and dhps. However, there are several advantages of the SSOP-ELISA. In general, the full haplotyping post-PCR of 88 samples in $d h f r$, dhps, or Pfcrt can be done within five hours. The results can be assessed visually or analyzed on an ELISA reader and can be exported into a computer spreadsheet format, providing a quick and objective analysis of the data. In contrast, dot blotting take two days and demands an expensive imager or manual analysis of the resulting dots. Finally, only half of the TMAC solution, which is a health hazard, is required per ELISA plate compared with dot blot membranes, thus reducing the amount of hazardous waste. 
The most promising practical application of the analyses for molecular markers of antimalarial drug resistance is determining the frequency of the relevant haplotypes in large-scale longitudinal studies as an adjunct to the laborious in vivo surveillance of drug resistance. This might provide data on the initial development and spread of antimalarial drug resistance prior to potential change of first-line treatment. Conversely, it may facilitate the surveillance of the possible restoration of drug susceptibility once drug pressure has ceased. As excellent examples of the latter, two recent studies performed in Malawi have shown that the prevalence of the codon 76 mutation in the Pfcrt gene and thus, the level of $P$. falciparum resistance to CQ has decreased markedly since the country abandoned the use of $\mathrm{CQ}$ as a first-line drug in $1993,{ }^{21,22}$ raising the intriguing possibility of reintroducing discarded drugs such as CQ. ${ }^{8}$ However, it is still controversial whether mutations in the Pfcrt gene are the sole mediator of $\mathrm{CQ}$ resistance and other alleles or loci may be involved. ${ }^{22}$ Furthermore, the prevalence of the wild-type $d h f r$ allele has been shown to increase as a result of installation of bed nets, possibly through decreased malaria transmission followed by less SP use. ${ }^{23}$

Monitoring molecular markers of drug resistance on a large scale in malaria-endemic areas demands a wide collection of samples collected routinely, as well as a simple method to perform high-throughput detection of the markers. Networks such as the East African Network for Monitoring Antimalarial Treatment (EANMAT) (www.eanmat.org) monitors annual sensitivity of $P$. falciparum to various antimalarial drugs at multiple sentinel sites in Kenya, Rwanda, Tanzania, Uganda, and Burundi. Thus, the network provides a unique basis for the measurement of molecular markers longitudinally that could be correlated with SP and CQ resistance and possibly other antimalarial drugs in the future. The SSOPELISA described here represents a suitable method to perform an analysis of such large numbers of samples within the countries involved.

The SSOP-ELISA may be extended to identify SNPs at other positions in $d h f r, d h p s$, or Pfcrt simply by designing new probes/primers. Furthermore, the technology can be modified into SNP analyses for other P. falciparum genes or in other disease-causing organisms.

Received July 14, 2004. Accepted for publication October 9, 2004.

Acknowledgments: We thank the staff of TPC Hospital, particularly Dr. H. Mwerinde for sample collection, and Jimmy Weng for excellent technical assistance in performing the PCR-ELISA and dot blotting. We also thank David Wallikers (University of Edinburgh, Edinburgh, United Kingdom) for providing $P$. falciparum isolates K1, 7G8, and DD2, and Trine Staalsoe and Kirsten Pihl (Centre for Medical Parasitology) for providing isolates 3D7 and FCR3.

Financial support: This study was supported by the Danish International Development Agency Research Council (RUF, grant no. 91106) and the Wellcome Trust (GR063516) and conducted as part of the Joint Malaria Programme, a collaboration between the National Institute for Medical Research, Kilimanjaro Christian Medical College, the London School of Hygiene and Tropical Medicine, and the Centre for Medical Parasitology, University of Copenhagen.

Authors' addresses: Michael Alifrangis, Insaf F Khalil, Anita M. Rønn, and Ib C. Bygbjerg, Centre for Medical Parasitology, Institute of Medical Microbiology and Immunology and Institute of Public Health, University of Copenhagen, Copenhagen, Denmark and Department of Infectious Diseases, Copenhagen University Hospital, Copenhagen, Denmark, E-mails: Alifrangis@cmp.dk, Insafk@ immu.ku.dk, ARN@euro-alarm.dk, and I.Bygbjerg@pubhealth. ku.dk. Sonia Enosse, Centre for Medical Parasitology, Institute of Medical Microbiology and Immunology and Institute of Public Health, University of Copenhagen, Copenhagen, Denmark and Instituto Nacional de Saúde, Ministério de Saúde, Maputo, Mozambique, E-mail: Senosse@hotmail.com. Richard Pearce and Cally Roper, Department of Infectious and Tropical Diseases, London School of Hygiene and Tropical Medicine, London WC1E 7HT, United Kingdom, E-mails: Richard.Pearce@lshtm.ac.uk and Cally.Roper@lshtm.ac.uk. Chris Drakeley, Department of Infectious and Tropical Diseases, London School of Hygiene and Tropical Medicine, London WC1E 7HT, United Kingdom and The Joint Malaria Programme, Moshi, Tanzania, E-mail: Chris.Drakely@ lshtm.ac.uk. Watoky M. M. M. Nkya, Kilimanjaro Christian Medical Centre, Moshi, Tanzania and The Joint Malaria Programme, Moshi, Tanzania, E-mail: Nkya10@hotmail.com. Thor G. Theander, The Joint Malaria Programme, Moshi, Tanzania, E-mail: Theander@ cmp.dk.

\section{REFERENCES}

1. The efficacy of antimalarial monotherapies, sulphadoxinepyrimethamine and amodiaquine in east Africa: implications for sub-regional policy, 2003. Trop Med Int Health 8: 860-867.

2. Kublin JG, Dzinjalamala FK, Kamwendo DD, Malkin EM, Cortese JF, Martino LM, Mukadam RA, Rogerson SJ, Lescano AG, Molyneux ME, Winstanley PA, Chimpeni P, Taylor TE, Plowe CV, 2002. Molecular markers for failure of sulfadoxinepyrimethamine and chlorproguanil-dapsone treatment of Plasmodium falciparum malaria. J Infect Dis 185: 380-388.

3. Kyabayinze D, Cattamanchi A, Kamya MR, Rosenthal PJ, Dorsey G, 2003. Validation of a simplified method for using molecular markers to predict sulfadoxine-pyrimethamine treatment failure in African children with falciparum malaria. Am J Trop Med Hyg 69: 247-252.

4. Omar SA, Adagu IS, Warhurst DC, 2001. Can pretreatment screening for dhps and dhfr point mutations in Plasmodium falciparum infections be used to predict sulfadoxinepyrimethamine treatment failure? Trans $\mathrm{R}$ Soc Trop Med Hyg 95: 315-319.

5. Fidock DA, Nomura T, Talley AK, Cooper RA, Dzekunov SM, Ferdig MT, Ursos LM, Sidhu AB, Naude B, Deitsch KW, Su XZ, Wootton JC, Roepe PD, Wellems TE, 2000. Mutations in the $P$. falciparum digestive vacuole transmembrane protein PfCRT and evidence for their role in chloroquine resistance. Mol Cell 6: 861-871.

6. Djimde A, Doumbo OK, Steketee RW, Plowe CV, 2001. Application of a molecular marker for surveillance of chloroquineresistant falciparum malaria. Lancet 358: 890-891.

7. Wellems TE, Plowe CV, 2001. Chloroquine-resistant malaria. J Infect Dis 184: 770-776.

8. Plowe CV, 2003. Monitoring antimalarial drug resistance: making the most of the tools at hand. J Exp Biol 206: 3745-3752.

9. Basco LK, Ndounga M, Tejiokem M, Ngane VF, Youmba JC, Ringwald P, Soula G, 2002. Molecular epidemiology of malaria in Cameroon. XI. Geographic distribution of Plasmodium falciparum isolates with dihydrofolate reductase gene mutations in southern and central Cameroon. Am J Trop Med Hyg 67: 378-382.

10. Duraisingh MT, Curtis J, Warhurst DC, 1998. Plasmodium falciparum: detection of polymorphisms in the dihydrofolate reductase and dihydropteroate synthetase genes by PCR and restriction digestion. Exp Parasitol 89: 1-8.

11. Alifrangis M, Enosse S, Khalil IF, Tarimo DS, Lemnge MM, Thompson R, Bygbjerg IC, Ronn AM, 2003. Prediction of Plasmodium falciparum resistance to sulfadoxine/pyrimethamine in vivo by mutations in the dihydrofolate reductase and dihydropteroate synthetase genes: a comparative study between sites of differing endemicity. Am J Trop Med Hyg 69: 601-606.

12. Pearce RJ, Drakeley C, Chandramohan D, Mosha F, Roper C, 2003. Molecular determination of point mutation haplotypes in the dihydrofolate reductase and dihydropteroate synthase of Plasmodium falciparum in three districts of northern Tanzania. Antimicrob Agents Chemother 47: 1347-1354.

13. Abdel-Muhsin AM, Ranford-Cartwright LC, Medani AR, 
Ahmed S, Suleiman S, Khan B, Hunt P, Walliker D, Babiker HA, 2002. Detection of mutations in the Plasmodium falciparum dihydrofolate reductase (dhfr) gene by dot-blot hybridization. Am J Trop Med Hyg 67: 24-27.

14. Mehlotra RK, Fujioka H, Roepe PD, Janneh O, Ursos LM, Jacobs-Lorena V, McNamara DT, Bockarie MJ, Kazura JW, Kyle DE, Fidock DA, Zimmerman PA, 2001. Evolution of a unique Plasmodium falciparum chloroquine-resistance phenotype in association with pfcrt polymorphism in Papua New Guinea and South America. Proc Natl Acad Sci U S A 98: 12689-12694.

15. Staalsoe T, Giha HA, Dodoo D, Theander TG, Hviid L, 1999. Detection of antibodies to variant antigens on Plasmodium falciparum-infected erythrocytes by flow cytometry. Cytometry 35: 329-336.

16. Sambrook J, Fritsch EF, Maniatis T, 1989. Molecular Cloning: A Laboratory Manual. Cold Spring Harbor, NY: Cold Spring Harbor Press.

17. Wooden J, Kyes S, Sibley CH, 1993. PCR and Strain Identification in Plasmodium falciparum. Parasitol Today 9: 303-305.

18. Djimde A, Doumbo OK, Cortese JF, Kayentao K, Doumbo S, Diourte Y, Dicko A, Su XZ, Nomura T, Fidock DA, Wellems TE, Plowe CV, Coulibaly D, 2001. A molecular marker for chloroquine-resistant falciparum malaria. $N$ Engl J Med 344: 257-263.
19. Conway DJ, Roper C, Oduola AM, Arnot DE, Kremsner PG, Grobusch MP, Curtis CF, Greenwood BM, 1999. High recombination rate in natural populations of Plasmodium falciparum. Proc Natl Acad Sci U S A 96: 4506-4511.

20. Hastings MD, Bates SJ, Blackstone EA, Monks SM, Mutabingwa TK, Sibley CH, 2002. Highly pyrimethamine-resistant alleles of dihydrofolate reductase in isolates of Plasmodium falciparum from Tanzania. Trans R Soc Trop Med Hyg 96: 674-676.

21. Kublin JG, Cortese JF, Njunju EM, Mukadam RA, Wirima JJ, Kazembe PN, Djimde AA, Kouriba B, Taylor TE, Plowe CV, 2003. Reemergence of chloroquine-sensitive Plasmodium falciparum malaria after cessation of chloroquine use in Malawi. J Infect Dis 187: 1870-1875.

22. Mita T, Kaneko A, Lum JK, Bwijo B, Takechi M, Zungu IL, Tsukahara T, Tanabe K, Kobayakawa T, Bjorkman A, 2003. Recovery of chloroquine sensitivity and low prevalence of the Plasmodium falciparum chloroquine resistance transporter gene mutation K76T following the discontinuance of chloroquine use in Malawi. Am J Trop Med Hyg 68: 413-415.

23. Alifrangis M, Lemnge MM, Ronn AM, Segeja MD, Magesa SM, Khalil IF, Bygbjerg IC, 2003. Increasing prevalence of wildtypes in the dihydrofolate reductase gene of Plasmodium falciparum in an area with high levels of sulfadoxine pyrimethamine resistance after introduction of treated bed nets. Am J Trop Med Hyg 69: 238-243.

APPENDIX 1

Prevalence of point mutations in $d h f r$ and dhps in Plasmodium falciparum isolated from malaria patients at the TPC sugarcane plantation in Moshi, Tanzania*

\begin{tabular}{|c|c|c|c|c|c|c|c|c|}
\hline & \multicolumn{4}{|c|}{$d h f r$} & \multicolumn{4}{|c|}{ dhps } \\
\hline & n & c51 & c59 & c108 & n & c436 & c437 & c540 \\
\hline \multirow{2}{*}{ wt $d h f r$} & \multirow{2}{*}{2} & & & & 1 & & & \\
\hline & & & & & 1 & & & \\
\hline \multirow{9}{*}{$\mathrm{db} d h f r$} & 1 & & & & 1 & & & \\
\hline & \multirow{3}{*}{3} & & & & 1 & & & \\
\hline & & & & & 1 & & & \\
\hline & & & & & 1 & & & \\
\hline & \multirow{5}{*}{9} & & & & 1 & & & \\
\hline & & & & & 1 & & & \\
\hline & & & & & 1 & & & \\
\hline & & & & & 1 & & & \\
\hline & & & & & 5 & & & \\
\hline \multirow{18}{*}{ tp $d h f r$} & 1 & & & & 1 & & & \\
\hline & \multirow{6}{*}{7} & & & & 1 & & & \\
\hline & & & & & 1 & & & \\
\hline & & & & & 1 & & & \\
\hline & & & & & 1 & & & \\
\hline & & & & & 1 & & & \\
\hline & & & & & 2 & & & \\
\hline & \multirow{3}{*}{8} & & & & 1 & & & \\
\hline & & & & & 1 & & & \\
\hline & & & & & 6 & & & \\
\hline & \multirow{8}{*}{39} & & & & 3 & & & \\
\hline & & & & & 2 & & & \\
\hline & & & & & 1 & & & \\
\hline & & & & & 1 & & & \\
\hline & & & & & 24 & & & \\
\hline & & & & & 1 & & & \\
\hline & & & & & 2 & & & \\
\hline & & & & & 5 & & & \\
\hline
\end{tabular}

